\title{
Papers
}

\section{Evaluation of three oligonucleotide primer sets in PCR for the identification of Burkholderia cepacia and their differentiation from Burkholderia gladioli}

Fiona E Clode, Mary E Kaufmann, Henry Malnick, Tyrone L Pitt

\begin{abstract}
Aims-To evaluate three oligonucleotide primer pairs-two specific for $16 S$ and $23 S$ rRNA sequences of Burkholderia cepacia, and the third specific for internal transcribed spacer region of $16 \mathrm{~S}-23 \mathrm{~S}$ sequences of $B$ gladioli-for the identification and differentiation of reference and clinical strains of these and other species.

Methods-The three primers sets were applied in polymerase chain reaction (PCR) to a collection of 177 clinical isolates submitted for identification from diagnostic laboratories as presumed $B$ cepacia.

Results-At an annealing temperature of $63^{\circ} \mathrm{C}$, all eight $B$ cepacia and four $B$ gladioli reference strains reacted with their specific primers. $B$ vandii was the only other species that was positive with both B cepacia primers but five Burkholderia or Ralstonia species reacted with one of these primers. Seventy eight isolates were typical of $B$ cepacia in biochemical tests and 75 of these reacted with specific primers; three, however, were positive with the $B$ gladioli primers. Fifteen asaccharolytic isolates were confirmed as $B$ cepacia by PCR but other non-fermenting Gram negative species were negative with each of the primers.

Conclusions-PCR using 16S rRNA sequences is recommended for identification of $B$ cepacia that give atypical results in biochemical tests. (F Clin Pathol 1999;52:173-176)

Keywords: PCR; B cepacia; cystic fibrosis.
\end{abstract} 11 November 1998

Table 1 Primer sets used for confirmation of B cepacia and B gladioli

\begin{tabular}{|c|c|c|c|c|}
\hline $\begin{array}{l}\text { Primer } \\
\text { set }\end{array}$ & Ref & Sequence & Target & $\begin{array}{l}\text { Amplicon } \\
\text { size (bp) }\end{array}$ \\
\hline P1 & $\begin{array}{l}\text { PC1 } \\
\text { PC2 }\end{array}$ & $\begin{array}{l}\text { 5'GCTGC GGATG CGTGC TTTGC 3' } \\
\text { 5'GCCTT CTCCA ATGCA GCGAC3' }\end{array}$ & $\begin{array}{l}\text { 23S rRNA } \\
\text { B cepacia }\end{array}$ & 323 \\
\hline P2 & $\begin{array}{l}\text { PSR1 } \\
\text { PSL1 }\end{array}$ & $\begin{array}{l}\text { 5'TTTCG AGCAC TCCCG CCTCT CAG3' } \\
\text { 5'AACTA GTTGT TGGGG ATTCA TTTC3' }\end{array}$ & $\begin{array}{l}16 \mathrm{~S} \text { rRNA } \\
\text { B cepacia }\end{array}$ & 209 \\
\hline P3 & $\begin{array}{l}\text { PG1 } \\
\text { PG2 }\end{array}$ & $\begin{array}{l}\text { 5'TTCAAT GACAA ACGTT CGGG 3' } \\
\text { 5'GCTTT CGCTT GACAG GCC 3' }\end{array}$ & $\begin{array}{l}\text { ITS rRNA } \\
\text { B gladioli }\end{array}$ & 274 \\
\hline
\end{tabular}

ITS, internal transcribed spacer.
Since the mid-1980s, Burkholderia (Pseudomonas) cepacia has been increasingly associated with respiratory infection in a minority of patients with cystic fibrosis. ${ }^{1}$ Some patients show little or no clinical decline following acquisition of the organism but others may succumb to a severe necrotising pneumonia, sometimes with septicaemia which is often rapidly fatal. This clinical problem is further compounded by the innate resistance of the species to many antibiotics and the demonstration of transfer of some strains from patient to patient. ${ }^{2}{ }^{3}$ As a consequence, many cystic fibrosis centres in Europe and North America segregate patients for treatment on the basis of their sputum bacteriology and advise patients with $B$ cepacia against social interaction with non-colonised patients.

The clinical and social implications of colonisation with $B$ cepacia make rapid and accurate identification of paramount importance. Most clinical laboratories use colistin containing selective media for the isolation and presumptive identification of $B$ cepacia but several colistin resistant environmental Gram negative species may grow on these media. ${ }^{4}$ Furthermore, traditional methods of identification may wrongly classify closely related spe-

Table 2 Reactions of reference strains of Burkholderia and Ralstonia with primer sets

\begin{tabular}{|c|c|c|c|c|}
\hline Species & Strain No & P1 & $P 2$ & $P 3$ \\
\hline B cepacia & NCTC 10661 & + & + & - \\
\hline B cepacia & NCTC 10744 & + & + & - \\
\hline B cepacia & ATCC 29424 & + & + & - \\
\hline B cepacia & ATCC 25608 & + & + & - \\
\hline B cepacia & ATCC 27515 & + & + & - \\
\hline B cepacia & ATCC 17460 & + & + & - \\
\hline B cepacia & ATCC 25610 & + & + & - \\
\hline B cepacia & ATCC 17765 & + & + & - \\
\hline B gladioli & NCTC 12378 & - & - & + \\
\hline B gladioli & ATCC 25417 & - & - & + \\
\hline B gladioli & ATCC 10247 & - & - & - \\
\hline B gladioli & ATCC 10248 & - & - & + \\
\hline B glumae & LMG 1277 & - & + & - \\
\hline B plantarii & LMG 10908 & - & - & - \\
\hline B vietnamiensis & LMG 6998 & + & - & - \\
\hline$B$ vandii & LMG 16020 & + & + & - \\
\hline$R$ solanacearum & LMG 2295 & + & - & - \\
\hline$B$ andropogonis & LMG 2126 & - & - & - \\
\hline B caryophylli & LMG 2155 & - & + & - \\
\hline$B$ cocovenenans & LMG 11626 & + & - & + \\
\hline R pickettii & NCTC 11149 & - & - & - \\
\hline Ps aeruginosa & NCTC 10332 & - & - & - \\
\hline
\end{tabular}

Primers as in table 1. 
Table 3 PCR reactions of presumptively identified " $B$ cepacia" with primer sets *

\begin{tabular}{lcrrrc}
\hline & $\begin{array}{l}\text { Number } \\
\text { of } \\
\text { isolates }\end{array}$ & $P 1$ & $P 2$ & P3 & $\begin{array}{l}\text { No } \\
\text { reaction }\end{array}$ \\
Biochemical identification & 78 & 75 & 75 & 3 & 0 \\
\hline Typical B cepacia & 15 & 15 & 15 & 0 & 0 \\
$\begin{array}{l}\text { Asaccharolytic B cepacia } \\
\text { "B cepacia" }\end{array}$ & 5 & 0 & 0 & 0 & 5 \\
B gladioli & 11 & 10 & 10 & 0 & 1 \\
Ps aeruginosa & 28 & 0 & 0 & 0 & 28 \\
$\begin{array}{l}\text { Other glucose } \\
\quad \text { non-fermenters }\end{array}$ & 24 & 0 & 0 & 0 & 24 \\
$\begin{array}{l}\text { Glucose fermenters } \\
\text { Not identified }\end{array}$ & 3 & 0 & 0 & 0 & 3 \\
Total & 13 & 0 & 0 & 0 & 13 \\
& 177 & 100 & 100 & 3 & 74
\end{tabular}

$\star$ Primer sets as in table 1 .

Isolates submitted for identification from diagnostic laboratories.

cies (for example, $B$ gladioli), as $B$ cepacia and many presumed $B$ cepacia cystic fibrosis isolates may give atypical biochemical reactions, making unequivocal identification difficult. Indeed, the Edinburgh epidemic strain of $B$ cepacia has been reported to have phenotypic features indicative of both $B$ cepacia and $B$ gladioli. ${ }^{5} 6$

Tyler et al targeted 23S rRNA sequences to generate oligonucleotide primers for amplification of a region specific for $B$ cepacia and used sequences from the internal transcribed spacer (ITS) region of $16 \mathrm{~S}-23 \mathrm{~S}$ rRNA for the identification of $B$ gladioli. ${ }^{7} B$ cepacia specific $16 \mathrm{~S}$ rRNA sequences were also exploited by Campbell et al for rapid polymerase chain reaction (PCR) identification of the species. ${ }^{8}$ We have evaluated sets of primers from the above studies on a number of biochemically typical and atypical isolates to determine their value for specific identification and differentiation of $B$ cepacia and $B$ gladioli from patients with cystic fibrosis.

\section{Methods}

BACTERIAL ISOLATES

The respective type strains of $B$ cepacia, $B$ gladioli, and Pseudomonas aeruginosa were obtained from the national collection of type cultures (NCTC) and the American type culture collection (ATCC). Other Burkholderia and Ralstonia species were provided by the LMG culture collection, Belgium. One hundred and seventy seven clinical isolates from cystic fibrosis patients were collected over 18 months and included those sent to the Laboratory of Hospital Infection for identification or typing. Environmental isolates of $B$ cepacia and some representative isolates of the "Edinburgh" epidemic strain of $B$ cepacia prevalent in the cystic fibrosis population, were kindly provided by Dr J R W Govan, University of Edinburgh Medical School, Edinburgh, UK.

BIOCHEMICAL IDENTIFICATION

All isolates were tested in the API 20NE gallery (BioMerieux) and examined for Gram stain reaction, motility, hydrolysis of casein, gelatin, starch, tyrosine, Tween 20 and Tween 80, production of DNase, catalase, oxidase, lecithinase, and growth on MacConkey, B cepacia selective media (Mast), and polyhydroxybutyrate agars.

\section{PRIMER SETS}

Oligonucleotide primer pairs were synthesised by Cruachem (Glasgow, UK) and the sequences are shown in table 1 .

POLYMERASE CHAIN REACTION

Crude bacterial DNA was prepared by emulsifying five colonies of 48 hours growth on nutrient agar in $100 \mu \mathrm{l}$ of sterile distilled water. After vortexing and centrifuging at $13000 \mathrm{~g}$ for five minutes, $3 \mu \mathrm{l}$ of the supernatant were added to $12 \mu \mathrm{l}$ of water. A water blank $(15 \mu \mathrm{l})$ was also prepared. The PCR mix contained $100 \mathrm{pmol}$ of each primer, $50 \mathrm{pmol}$ of $\mathrm{MgCl}_{2}, 2.5 \mathrm{pmol}$ of each of the deoxynucleotide triphosphates, 1.25 units of Taq DNA polymerase, and $2.5 \mu \mathrm{l}$ of 10× PCR buffer (Life Technologies). In the multiplex PCR, each extra primer used replaced $1 \mu l$ of water.

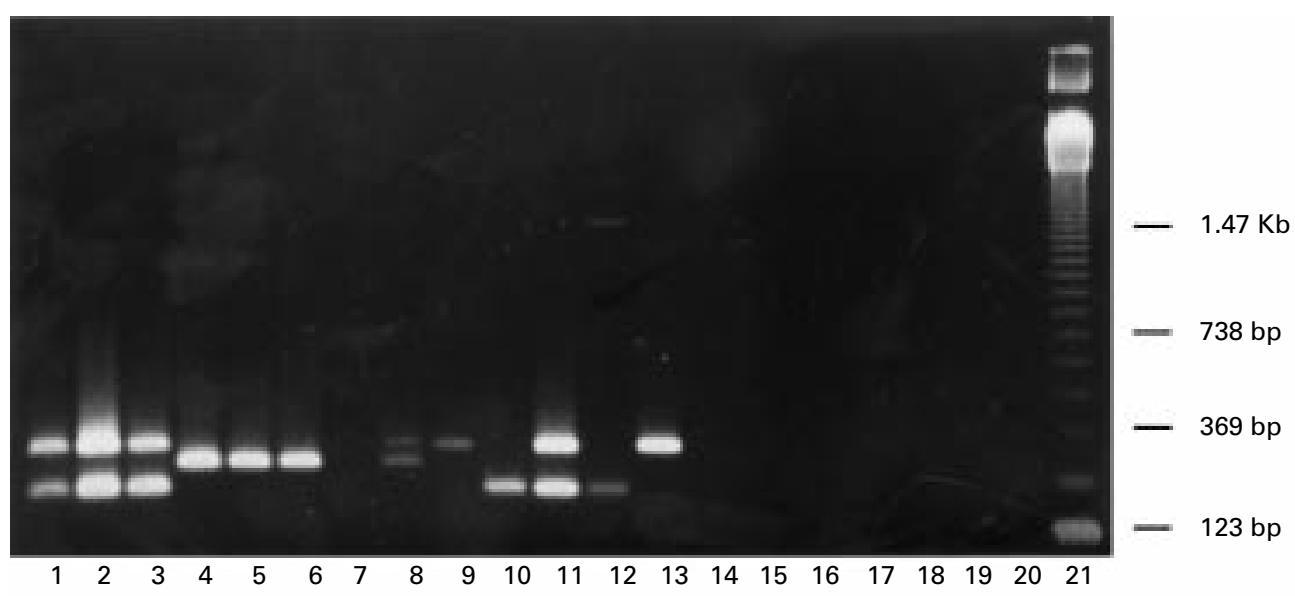

Figure 1 Multiplex PCR with three primers (see table 1) and reference strains of Burkholderia spp and other species. Lane 1: B cepacia NCTC10661; lane 2: B cepacia NCTC10744; lane 3: B cepacia ATCC25610; lane 4: B gladioli NCTC12378; lane 5: B gladioli ATCC10248; lane 6: B gladioli ATCC25417; lane 7: R pickettii NCTC11149; lane 8: B cocovenenans LMG11626; lane 9: R solanacearum LMG2295; lane 10: B caryophylli LMG2155; lane 11: B vandii LMG16020; lane 12: B glumae LMG1277; lane 13: B vietnamiensis LMG6998; lane 14: B plantarii LMG10908; lane 15: B andropogonis LMG2126; lane 16: Ps aeruginosa NCTC10332; lane 17: Ps aeruginosa NCTC10662; lane 18: Acinetobacter baumannii; lane 19: S maltophilia; lane 20: water blank; lane 21: 123 bp size markers. 
Amplification was carried out in a Thermal cycler (Hybaid) for 25 cycles. DNA was denatured at $96^{\circ} \mathrm{C}$ for five minutes for one cycle, and at $96^{\circ} \mathrm{C}$ for 15 seconds for cycles 2 to 25 . The optimum annealing conditions were determined between temperatures of $58^{\circ} \mathrm{C}$ and $63^{\circ} \mathrm{C}$ for 30 seconds and primer extension was at $72^{\circ} \mathrm{C}$ for 90 seconds. An additional primer extension was carried out at $70^{\circ} \mathrm{C}$ for five minutes. PCR products were separated in a $1.5 \%$ Nusieve agarose gel (Flowgen) at $100 \mathrm{~V}$ for 1.5 hours and molecular weights were determined by comparison with a $123 \mathrm{bp}$ ladder (Life Technologies).

\section{Results}

The reactions of the Burkholderia and Ralstonia reference strains and Ps aeruginosa, with the three sets of primers, are shown in table 2 and illustrated in fig 1 as a multiplex PCR. The optimal annealing temperature for each of the primers was $63^{\circ} \mathrm{C}$ and all $\mathrm{B}$ cepacia reference strains produced amplicons with both sets of homologous primers, but not with the $B$ gladioli primers and vice versa. However, one reference strain of $B$ gladioli failed to react with primer P3. Four other Burkholderia spp and the $R$ solanacearum reference strain were positive in the PCR with either primer P1 or P2 but $B$ vandii reacted with both. $B$ andropogonis, $B$ plantarii, and $R$ pickettii were negative with each of the three primer sets.

Over 18 months, 177 isolates were submitted for species confirmation by clinical diagnostic laboratories as presumed $B$ cepacia from cystic fibrosis patients and most isolates grew on the selective medium. Table 3 summarises the results of the PCR with the three primer pairs and shows that 100 isolates reacted with primers P1 and P2. Of these, 75 were typical of $B$ cepacia in biochemical tests, 15 were asaccharolytic, and 10 gave biochemical reactions most consistent with an identification of $B$ gladioli. None of the latter isolates formed products with primer P3. However, this primer reacted with three biochemically typical $\mathrm{B}$ cepacia isolates. Five clinical isolates which were negative in the PCR, grew on the selective medium, and were classified by colonial morphology, pigment, and other phenotypic tests as closest to " $B$ cepacia" although the API $20 \mathrm{NE}$ classified them as "doubtful profile" for the species. The 24 other glucose nonfermenters included alkaline forming pseudomonads and Stenotrophomonas maltophilia; 28 strains proved to be Ps aeruginosa and three were found to ferment glucose. None of these reacted in the PCR. Ten environmental isolates of $B$ cepacia from the Edinburgh Botanical Garden were confirmed by PCR as was the Edinburgh epidemic strain.

\section{Discussion}

Yabuuchi et al proposed that seven species formerly of pseudomonas RNA group II should be transferred to a new genus, Burkholderia, with $B$ cepacia as the type species. ${ }^{9}$ The genus included $B$ caryophylli, $B$ gladioli, $B$ mallei, $B$ pseudomallei, $R$ pickettii, and $R$ solanacearum. Recently, the latter two species have been transferred to the genus Ralstonia. ${ }^{10}$ Two species, $B$ plantarii and $B$ glumae, were added to the genus on the basis of phenotypic and chemotaxonomic characteristics ${ }^{11}$ and they were later joined by $B$ vandii and $B$ cocovenenans. ${ }^{12}$ Gillis et al described nitrogen fixing strains of the same rRNA complex as $B$ cepacia and named them $B$ vietnamiensis because of their isolation from macerates of rice cultured in Vietnam. ${ }^{13}$ Two further pseudomonads (Ps glathei and Ps pyrrocinia) have been proposed for reclassification as Burkholderia. ${ }^{14}$

A selective and differential medium $^{15}$ is widely used in clinical laboratories for the culture of $B$ cepacia from cystic fibrosis sputum, but identification of the species remains problematic. Holmes ${ }^{16}$ found that $4 \%$ of Gram negative non-glucose fermenting strains sent to the NCTC for identification were $B$ cepacia, which suggested that the species occurs regularly in clinical material and that diagnostic laboratories have difficulty in identifying it. Indeed, the five genomic species recently recognised by Vandamme $e a^{14}$ include strains with asaccharolytic or atypical characteristics that API 20NE fails to identify to confident levels. API 20NE also fails to distinguish between $B$ cepacia and $B$ gladioli. The latter has been isolated from the cystic fibrosis lung and is generally considered to be more of a hindrance to the identification of $B$ cepacia than a pathogen in its own right. ${ }^{5}{ }^{17}$ However, a fatal empyema and bloodstream infection was reported in a patient with cystic fibrosis following lung transplantation. ${ }^{18}$

The three primer pairs tested here were equally sensitive and specific, but for $B$ cepacia P2 was preferred for routine use as the product band was invariably clearer than with $\mathrm{P} 1$. The absence of reaction by any of the primers with the $R$ pickettii type strain or $S$ maltophilia is noteworthy as these organisms may on occasion colonise the cystic fibrosis lung. Indeed, Burdge et al described three instances of the latter species being misidentified as $B$ cepacia in sputum from cystic fibrosis patients. ${ }^{19}$ In each case the organisms grew well on selective medium and were initially incorrectly characterised as oxidase positive and DNase negative. Of the strains examined here, fewer than $50 \%$ were biochemically typical $B$ cepacia and so the confirmation by PCR was essential. It was unexpected to receive Ps aeruginosa isolates as presumed $B$ cepacia as the former are invariably sensitive to colistin in the selective medium. This may have been because of incorrect colony picks from non-selective media or because colistin resistant Ps aeruginosa, albeit rare, occur in cystic fibrosis patients. The five "B cepacia" strains may represent genomic groups of $B$ cepacia different from those detected with the primers used here and further investigation in this area is warranted.

In summary, the perception and consequences of $B$ cepacia colonisation by cystic fibrosis patients and their carers makes it of paramount importance to have unequivocal identification of the species. The PCR method described here is rapid, sensitive and specific in 
the light of current taxonomic classification. It is therefore recommended for the confirmation of the identity of isolates presumptively classified as $B$ cepacia by biochemical tests.

We thank Dr Wendy Johnson and Shaun Tyler for providing us with sequences and advice before publication of their paper.

1 Govan J, Hughes J, Vandamme P. Burkholderia cepacia: medical, taxonomic and ecological issues. 7 Med Microbio medical, taxonomic

2 LiPuma J, Dasen S, Nielson D, et al. Person-to-person transmission of Pseudomonas cepacia between patients with cystic fibrosis. Lancet 1990;336:1094-6.

3 Govan JRW, Brown PH, Maddison J, et al. Evidence for transmission of Pseudomonas cepacia by social contact in cystic fibrosis. Lancet 1993;342:15-19.

4 Hutchinson G, Parker S, Pryor J, et al. Home-use nebulizers: a potential primary source of Burkholderia cepacia and other colistin resistant, Gram-negative bacteria in patients with cystic fibrosis. F Clin Microbiol 1996;34: 584-7.

5 Simpson I, Finlay J, Winstanley D, et al. Multi-resistance isolates possessing characteristics of both Burkholderia (Pseudomonas) cepacia and Burkholderia gladioli from patients with cystic fibrosis. I Antimicrob Chemother patients with cyst

6 Baxter I, Lambert P, Simpson I. Isolation from clinical sources of Burkholderia cepacia possessing characteristics of Burkholderia gladioli. $\mathcal{F}$ Antimicrob Chemother 1997;39: 169-75.

7 Tyler S, Strathdee C, Rozee K, et al. Oligonucleotide primers designed to differentiate pathogenic pseudomonads on the basis of the sequencing of genes coding for $16 \mathrm{~S}-23 \mathrm{~S}$ rRNA internal transcribed spacers. Clin Diagn Lab Immunol 1995;2:448-53.

8 Campbell PW, Phillips JA, Heidecker GJ, et al. Detection of Pseudomonas (Burkholderia) cepacia using PCR. Pediatr Pulmonol 1995;20:44-9.

9 Yabuuchi E, Kosako Y, Oyaizu $\mathrm{H}$, et al. Proposal of Burkholderia gen. nov. and transfer of seven species of the genus Pseudomonas homology group II to the new genus with the type species Burkholderia cepacia (Palleroni and Holmes 1981) comb. nov. Microbiol Immunol 1992;36:897904.
10 Yabuuchi E, Kosako Y, Yano I, et al. Transfer of two Burkholderia and an Alcaligenes species to Ralstonia gen. nov.: proposal of Ralstonia pickettii (Ralston, Palleroni and Doudoroff 1973) comb. nov. Ralstonia solanacearum (Smith 1996) comb. nov. and Ralstonia eutropha (Davis 1969) comb. nov. Microbiol Immunol 1995;39:897-904.

11 Urakami T, Ito-Yoshida C, Aaraki H, et al. Transfer of Pseudomonas plantarii and Pseudomonas glumae to Burkholderia as Burkholderia spp. and description of Burkholderia vandii sp. nov. Int $\mathcal{F}$ Syst Bacteriol 1994;44:235-45.

12 Zhao N, Qu C, Wang E, et al. Phylogenetic evidence for the transfer of Pseudomonas cocovenenans (Vandamme et al. $1960)$ to the genus Burkholderia as Burkholderia cocovenenans (Vandamme et al. 1960) comb. nov. Int $\mathcal{F}$ Syst Bacteriol 1995;45:600-3.

13 Gillis M, Van T, Bardin R, et al. Polyphasic taxonomy in the genus Burkholderia leading to an amended description of the genus and proposition of Burkholderia vietnamiensis sp. nov. for N2-fixing isolates from rice in Vietnam. Int $\mathcal{f}$ Syst Bacteriol 1995;45:274-89.

14 Vandamme P, Holmes B, VanCanneyt M, et al. Occurrence of multiple genomovars of Burkholderia cepacia in cystic fibrosis patients; Proposal of Burkholderia multivorans sp. nov. Int f Syst Bacteriol 1997;47:1188-200.

15 Welch D, Muszynski M, Pai C, et al. Selective and differential medium for recovery of Pseudomonas cepacia from the respiratory tracts of patients with cystic fibrosis. 7 Clin Microbiol 1987;25:1730-4.

16 Holmes B. The identification of Pseudomonas cepacia and its occurrence in clinical material. F Appl Bacteriol 1986;61: 299-314.

17 Christenson J, Welch D, Mukwaya G, et al. Recovery of Pseudomonas gladioli from respiratory tract specimens of patients with cystic fibrosis. F Clin Microbiol 1989;27: 270-3.

18 Khan S, Gordon S, Stillwell $\mathrm{P}$, et al. Empyema and bloodstream infection caused by Burkholderia gladioli in a patient with cystic fibrosis after lung transplantation. Pediatr Infect Dis f 1996;15:637-9.

19 Burdge D, Noble M, Campbell M, et al. Xanthomonas maltophilia misidentified as Pseudomonas cepacia in cultures of sputum from patients with cystic fibrosis: a diagnostic pitfall with major clinical implications. Clin Infect Dis 1995; 20:445-8. 